\title{
Saturation Effects in the Phonon Reflection at a Quartz-Liquid Helium Interface*
}

\author{
H. Schubert, P. Leiderer, and H. Kinder \\ Physik-Department E 10, Technische Universität München, Garching, West Germany
}

|Received October 16, 1979|

\begin{abstract}
The reflection coefficient of longitudinal $25-\mathrm{GHz}$ phonons at a quartz-liquid ${ }^{4} \mathrm{He}$ interface was measured for various phonon intensities. For thoroughly cleaned quartz surfaces a reflection coefficient of 1 independent of the incident intensity was found. When the sample was covered with a thin hydrocarbon film, however, the reflection coefficient dropped by typically 5-10\% when the phonon intensity was low. At high intensities this extra phonon loss disappeared, suggesting the saturation of the inelastic processes at the quartzhelium interface.
\end{abstract}

\section{INTRODUCTION}

The heat transport from a solid into liquid helium is surprisingly effective in view of the "classical" mechanism of phonon transmission. Therefore, an additional mechanism is generally assumed, which is called anomalous Kapitza conductance (for a review see, e.g., Ref. 1). This mechanism is already operative when there is only a thin film of 1-2 atomic layers on the surface. This is demonstrated by the fact that a thin film can absorb monochromatic phonons and, after a delay time, reemit phonons with shifted frequencies back into the solid. ${ }^{2}$ Thus, the additional mechanism must be related to the properties of these 1-2 atomic layers, which are known to be strongly attracted by the solid through van der Waals forces.

One remarkable property of the film is in fact the strong anharmonicity which manifests itself by the phonon frequency shifts. One might relate this to a possible disordered structure at the surface. That disordered materials exhibit strong anharmonicities indeed was recently observed in glasses. ${ }^{3}$ So it is natural to look for other properties that are characteristic for a

*Based on a doctoral thesis submitted by $\mathrm{H}$. Schubert in partial fulfilment of the requirements for the degree Dr. rer. nat. at the Technische Universität München. 
disordered structure. The most pronounced property of glasses, which has led to the picture of two-level systems, ${ }^{4}$ is that of the saturation of the ultrasonic absorption at higher power levels. The purpose of this paper is to look for similar properties of the helium film, i.e., for a saturation of the anomalous Kapitza conductance at high power levels of monochromatic phonons.

To reach saturation, the phonons should not only be intense, but also highly monochromatic. Therefore, stimulated Brillouin scattering appears as the most promising method.

\section{EXPERIMENTAL}

Stimulated Brillouin scattering (SBS) has been used in the past as a convenient method to generate and investigate phonons in the $\mathrm{GHz}$ range (for a review see, e.g., Ref. 5). The setup in general is quite simple: to achieve SBS the intense beam of a giant pulse laser is focused into a sample. Above a certain threshold intensity the interaction between this incident light (together with backscattered Brillouin light) and the sample material leads to the buildup of a very intense longitudinal phonon field. These phonons are highly monochromatic, just as the incident laser light, and have a wavelength given by

$$
\lambda_{\mathrm{ph}}=\lambda_{L} / 2 n
$$

where $\lambda_{L}$ is the laser wavelength, e.g., $694.3 \mathrm{~nm}$ for a ruby laser, and $n$ is the refractive index of the material. The resulting phonon wavelength in a typical material like X quartz is $224 \mathrm{~nm}$, which corresponds to a frequency of $24.8 \mathrm{GHz}$. In addition to the phonons which propagate in the direction of the incident light, an intense beam of backscattered Brillouin light is observed, shifted in frequency by

$$
\Delta \nu=2 n v_{s} / \lambda_{L}
$$

$\Delta \nu$ is equal to the frequency of the SBS phonons; thus an analysis of the Brillouin light can be used, according to (2), to determine the phonon velocity $v_{s}$.

Important modifications were necessary for the application of SBS to the problem of the Kapitza resistance. Since the phonon reflection coefficient at the solid surface was to be determined to a high accuracy, plans were to use a thin sample and observe a large number of successive reflections of the SBS phonons. On the other hand, for the stimulation process a large interaction volume between laser light and sample and hence a thick sample is required. This dilemma was circumvented with the setup shown in Fig. 1. 


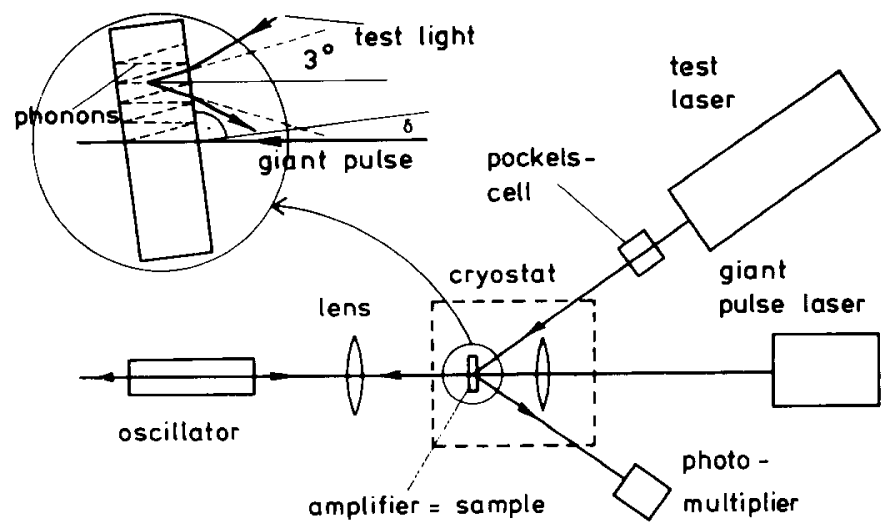

Fig. 1. Experimental setup for measuring the reflection coefficient of phonons at a solid-liquid helium interface. The phonons are generated in the sample by superposition of the light of the giant pulse laser and the SBS light from the oscillator. Phonon detection is achieved by Bragg scattering of the test laser beam (Bragg angle $3^{\circ}$ ). The inset shows the phonon path in a sample tilted by an angle $\delta$. The width of the phonon beam as well as the diameter of the focused test light are about $0.2 \mathrm{~mm}$.

The method is derived from the oscillator-amplifier technique used by Pohl et al. ${ }^{6}$ For the generation of phonons, light from a giant pulse ruby laser (pulse length $20 \mathrm{nsec}$, peak power $5 \mathrm{MW}$ ) is focused into a quartz crystal, which serves as "oscillator." This crystal has a length sufficient for the stimulation process to occur. The only task of the oscillator is to supply an intense beam of Brillouin light. Superposition of Brillouin and laser pulse in the "amplifier," which is the actual sample, then leads to the generation of coherent phonons as for usual SBS; the difference, however, is that for this process no threshold exists, so that the sample, a quartz plate, can be made very thin.

The fact that a threshold does not exist also allows us to vary the intensity of the phonons simply by detuning the Brillouin frequency of the oscillator with respect to that of the amplifier. This was achieved by slightly changing the orientation of the oscillator crystal, making use of the anisotropy of the sound velocity.

The phonons generated in this way can be detected with another laser beam, which is Bragg-reflected from the coherent phonon field. The resonator length of this second ruby laser was increased to several meters, and its $Q$-switch was removed. The emitted light pulses then have an intensity a factor of 100 below that of the giant pulse laser, but are considerably longer, and are thus suitable to probe the phonon field long after the generation process has ended. In the experiment, the light of this 
test laser was switched on by means of a Pockels cell only after the phonons had been generated by the giant pulse to avoid heating of the sample.

The Bragg-scattered light was detected by a photomultiplier, and the pulse pattern of each single shot was displayed on a fast storage oscilloscope. Simultaneously the intensity of the incident test laser light, which was slightly varying in time, was recorded on a second oscilloscope. The ratio of the scattered and the incident light intensity is then proportional to the phonon intensity.

Since the intensity of the Bragg-scattered test light is weak, the strong SBS background has to be separated from it in order not to disturb the measurement. This was achieved in the following way: The ruby of the test light laser was cooled to liquid nitrogen temperature, where laser emission is shifted from 694.3 to $693.4 \mathrm{~nm}$. The Bragg angle for the test light is then $3^{\circ}$. This allows a spatial separation from the SBS beam. Still, a certain amount of SBS light, and also of incident laser light, is scattered from imperfections of the sample into the direction of the photomultiplier. This component at $694.3 \mathrm{~nm}$ was suppressed by an interference filter.

The test light reflected from the sample consists of two parts, the actual signal due to the backscattering from the phonons, and a background beam reflected from the sample surface. In order to reduce the latter component, the sample plane was tilted slightly so that the geometrically reflected beam could be blocked off. Due to the inclination of the quartz plate by an angle $\delta$ with respect to the giant pulse beam the SBS phonons, after being reflected once from the sample surface, do not meet the Bragg condition for the test light. Only after the second (and subsequent even) reflections, when the phonons propagate in their original direction, is Bragg-scattered light to be expected. This leads to the maximum-minimum structure shown in Figs. 2 and 3.

Tilting the sample plane has an additional advantage: After every second reflection the position of the phonon beam is shifted by a small amount $\Delta a=\delta \cdot d$, where $d$ is the thickness of the sample. Therefore the phonons will have left their generation volume after a certain time, adjustable by changing the angle $\delta$, and can then be probed in a crystal region not directly exposed to the high intensity of the giant pulse. Regarding possible heating effects, this check appears to be very important.

\section{RESULTS}

Figure 2 shows a typical signal obtained from a quartz plate $0.35 \mathrm{~mm}$ thick and suspended in vacuum, i.e., without helium at the surface. In this example the test light completely overlapped the phonon generation volume, and the tilt angle of the plate was so small that the phonons could 


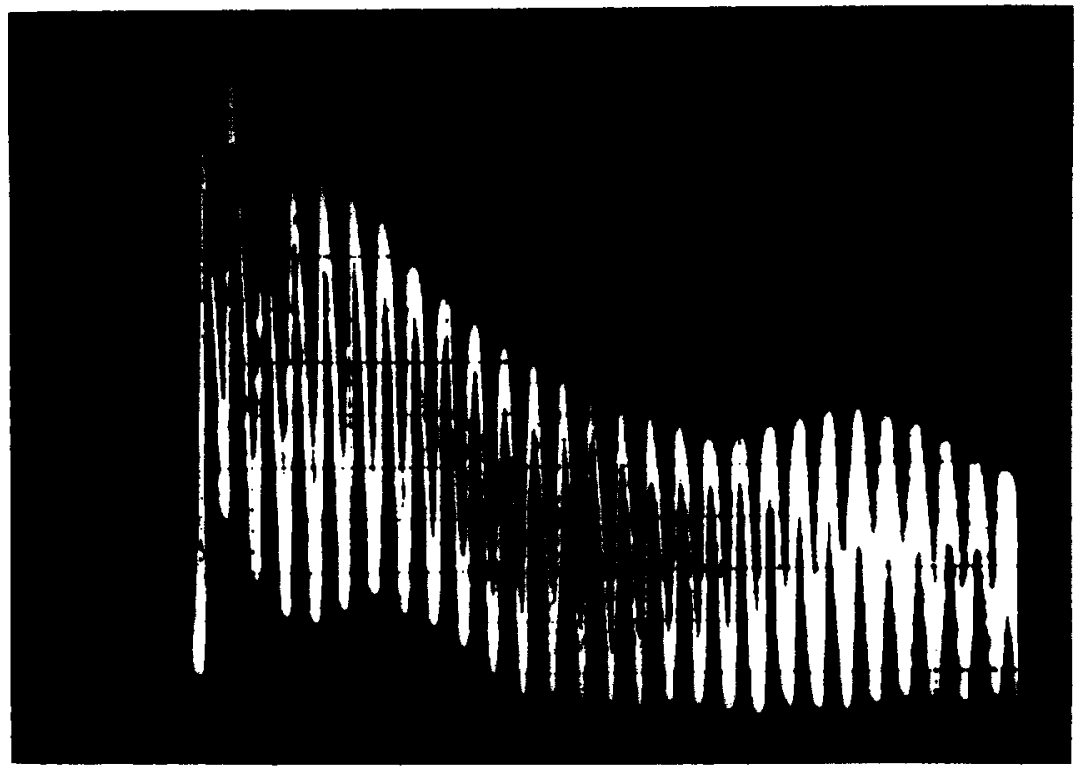

Fig. 2. Light intensity as obtained from Bragg scattering at coherent longitudinal phonons in a $0.35-\mathrm{mm}$-thick X-quartz plate. The echo pattern results from successive reflections of the phonons at the sample surface. The time interval between the peaks, corresponding to one round trip of the phonons, is $110 \mathrm{nsec}$. The slightly oscillating structure is due to the varying intensity of the incident test light. The sample, suspended in vacuum, was at a temperature of $1.8 \mathrm{~K}$.

not leave the test light volume during the displayed time interval of $3 \mu \mathrm{sec}$. The first pulse on the oscilloscope trace, a superposition of stray light from the giant pulse and of Bragg-scattered light, marks the time of phonon generation. The subsequent pulse pattern arises from phonons successively reflected back and forth in the sample plate. The distance between the pulse maxima, $110 \mathrm{nsec}$, corresponds to one round trip of the phonons.

A decay of the pulse heights on Fig. 2 is only barely visible, which indicates that even after 50 reflections the number of phonons is nearly the same. This implies not only that phonon damping in the bulk material is very low-which is known already from other experiments ${ }^{7}$ - but also that the phonon reflection coefficient at the surface is close to unity. Loss of phonons due to inelastic processes is therefore negligible when no helium is present.

In Fig. 3 a similar pulse train is shown, yet with the sample plate tilted at $\delta=2^{\circ}$, and with the focus of the test light shifted $0.5 \mathrm{~mm}$ away from the phonon generation volume. Consequently the SBS phonons could not be detected immediately after the giant pulse. The Bragg signal only appeared after about 20 phonon reflections, increased to a maximum, when the phonon field was completely within the test light volume, and then decayed 


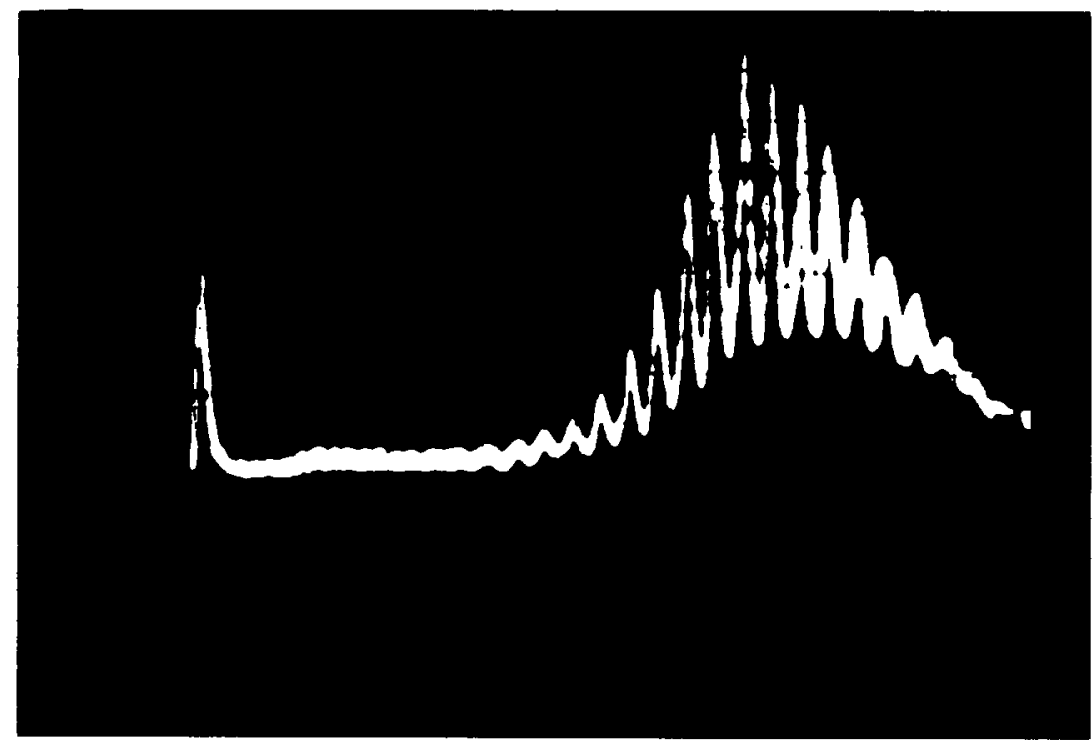

Fig. 3. Echo pattern obtained under similar conditions as in Fig. 2, but with the tilt angle $\delta$ increased and the detection volume shifted, so that the phonons become observable only after about 20 reflections.

again as the phonons were gradually leaving that volume. Although an absolute determination of the reflection coefficient $R$ was not possible in this case, the change of $R$ by helium could still be accurately determined by the following procedure: First, the pulse heights of each pattern were normalized with respect to the incident test light as described above. Then, an average over 15 similar patterns was taken with the sample in helium, and, in the same way, also with the sample in vacuum. The normalized and averaged pulse heights with helium were then divided by the pulse heights without helium. This yields the loss of phonon intensity from pulse to pulse due to the presence of helium, i.e., the change of $R$, by noting that the number of reflections is twice the pulse number. The resulting accuracy in $R$ was better than $\pm 1 \%$.

In preliminary measurements we found that when the quartz surface was covered with helium a reflection coefficient of less than 1 was observed indeed. As expected from the measurements of Sabinsky and Anderson, ${ }^{8}$ however, this helium effect at $25 \mathrm{GHz}$ was crucially dependent on the surface treatment. When the sample was carefully cleaned before the experiment and cold traps were used to shield the sample surface from pump oil vapors, the reflection coefficient was not affected by the presence of helium within our accuracy. Under less controlled conditions, on the other hand, the decrease in $R$ by addition of helium usually amounted to $5-10 \%$. 


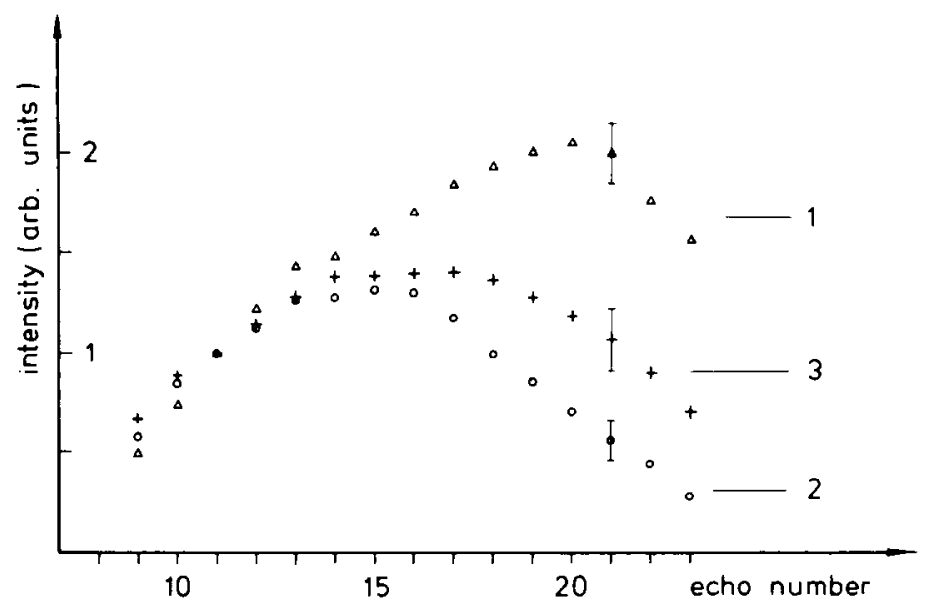

Fig. 4. Echo intensity averaged over 15 patterns of the type shown in Fig. 3 and arbitrarily normalized to echo number 11 . The phonon frequency is $24.8 \mathrm{GHz}$, the sample temperature $1.8 \mathrm{~K}$. (1) Sample on vacuum; $(2,3)$ sample in liquid ${ }^{4} \mathrm{He}$. The phonon intensity for data set 3 is about five times higher than for set 2 . The absolute value of the intensity, not determined to a high accuracy, is of the order of $10 \mathrm{~W} / \mathrm{cm}^{2}$.

Therefore in the following experiments a definite amount of impurities was used by covering the thoroughly cleaned sample on both sides with a layer, $100-1000 \AA$ thick, consisting of a mixture of alcanes with carbon atoms ranging between 8 and 16 .

Figure 4 shows echo intensities for a sample treated in such a way (the alignment of the sample plane and the test light beam corresponds to that of Fig. 3). For pulse sequence 1, the quartz plate was in vacuum, while for 2 and 3 it was immersed in bulk liquid helium. In order to detect possible saturation effects, the phonon intensity was varied, and for the data of sequence 3 was chosen to be a factor of five higher than for sequence 2 .

Because of the different phonon intensities, the pulse heights are arbitrarily normalized at echo number 11 for a better comparison of the envelope shape. All three echo patterns were obtained during the same experimental run, and from the same spot of the sample surface, so that also the details of the hydrocarbon layer were always the same.

Two conclusions can be drawn from Fig. 4:

(i) From sequence 2 it is obvious that adding helium to the surface leads to a distinctive reduction of the phonon reflection coefficient. This indicates that a hydrocarbon film and helium, simultaneously present, effectively attenuate phonons incident on the surface.

(ii) A comparison of sequences 2 and 3 shows that the reflection coefficient depends on the incident phonon intensity. Apparently, $R$ is 
higher for larger intensity, which is typical for a saturation behavior. The value of $R$ derived from the normalized echo amplitudes is $R=0.94$ for the low and $R=0.97$ for the high intensity.

On several individual shots, the phonon intensity was even large enough so that the reflection coefficient was indistinguishable from $R=1.0$, indicating complete saturation.

Before we can actually interpret our results in terms of saturation effects, however, we must carefully consider more trivial explanations.

(i) Most obviously, the intense phonon beam might remove the helium atoms from the sample surface by excessive evaporation. ${ }^{9}$ This would increase the reflection coefficient toward the value in vacuum. As a consequence, the evaporating He atoms should form a gas film on the sample. One should be able to detect this film by optical means as soon as its thickness becomes comparable to the wavelength of the test light. In a separate experiment, we have therefore generated heat pulses by the absorption of light in the sample, and we found that such a gas bubble can develop indeed. However, for the bubble to appear within $500 \mathrm{nsec}$, a phonon intensity of the order of $1000 \mathrm{~W} / \mathrm{cm}^{2}$ incident on the sample surface was required. When the intensity was reduced by a factor of five, no bubble formation was observed. Since in the present experiment the maximum phonon intensity was only about $10 \mathrm{~W} / \mathrm{cm}^{2}$, and more than $90 \%$ of the phonons were reflected, our intensity was by far not sufficient to evaporate $\mathrm{He}$ atoms in the presence of bulk liquid.

(ii) The direct interaction of the giant pulse and Brillouin light with the surface, on the other hand, cannot be responsible for the increase in $R$ either. First, the generation and detection volumes were separated in the experiment shown in Fig. 4. Second, the phonon intensity was changed by detuning the Brillouin frequencies (see above), so that the light intensities were always constant except for unavoidable fluctiations $(5 \%)$ of the laser. To study the influence of these fluctuations we varied in one run the light intensity on purpose by a factor of two while keeping the phonon intensity constant. The reflection coefficient remained unchanged.

(iii) We should also mention that a variation of the phonon intensity with the sample in vacuum did not show any influence on the reflection coefficient.

As already mentioned, also for samples prepared in a less well-defined way-namely those exposed to pump oil vapors $-R$ was found to drop from the vacuum value $R=1.0$ by typically $5-10 \%$ when helium was admitted, and sometimes reflection coefficients even as low as $R=0.8$ were observed. The phonon loss at the surface could again be saturated almost completely by high acoustic intensity.

For saturation effects to appear it was not necessary that the quartz plate was surrounded actually by bulk liquid. Measurements in helium gas 
yielded the same results even when the sample was covered with a film of only about two atomic layers. This further indicates that the processes responsible for the observations reported here must be located in the immediate vicinity of the quartz-helium interface.

\section{DISCUSSION}

Our results confirm that there exists a discrepancy between the theoretical and the experimental phonon reflection coefficient for a frequency between the usual ultrasonic regime-where so far no anomalous reduction in $R$ has been observed-and the high frequencies of heat pulse and phonon spectroscopy techniques. In agreement with results of Sabisky and Anderson, ${ }^{8}$ the decrease of the reflection coefficient in the present measurements at $25 \mathrm{GHz}$ is not as pronounced as for frequencies above $100 \mathrm{GHz}$.

In our experiment a relatively simple cleaning procedure-soaking the samples in highly pure acetone and distilled water-was sufficient to suppress the anomaly. This compares well with the results of Anderson and Johnson, ${ }^{10}$ who observed a Kapitza conductance close to the Khalatnikov value (which corresponds to a large reflection coefficient) on only midly cleaned samples for long-wavelength thermal phonons in the low-GHz range. In comparison, at a phonon frequency of $280 \mathrm{GHz}$, Weber et al. ${ }^{11}$ had to cleave crystals at low temperatures and in ultrahigh vacuum in order to prepare surfaces clean and smooth enough so that a reflection coefficient close to 1 could be obtained. The difference in the sensitivity to surface treatment might be attributable to the phonon wavelength, which in the present work is an order of magnitude larger than in Weber et al.'s experiment. It seems plausible that irregularities at the surface, as long as they are small, can affect only short-wavelength phonons, whereas a deeper roughness or larger amounts of impurities are required to affect the $200 \mathrm{~nm}$ phonons investigated here.

What appears as the most important aspect of our results is that saturation of the phonon losses at the surface can be achieved by high acoustic intensity. This feature bears resemblance with the acoustic properties of amorphous solids like glasses, where the saturation of ultrasonic absorption has been studied quite thoroughly. ${ }^{12}$ In these materials the attenuation of phonons is ascribed in part to resonant absorption of twolevel systems, or tunneling states, resulting from the disordered structure. At sufficiently high intensities, these two-level systems are equally populated and then can no longer contribute to the absorption.

In our experiment a similar explanation for the intensity dependence of the phonon reflection coefficient is suggestive. As for most other disordered systems studied to date, the microscopic origin of the two-level systems is 
somewhat obscure. Yet one might think of two possible mechanisms for how the saturable phonon loss at the surface can arise:

(i) A sufficient number of two-level systems might exist in the disordered hydrocarbon chains already without helium. In this case the lifetime of the excited states must be assumed to be long enough so that saturation is reached even with our lowest intensities. Without helium one will therefore observe a reflection coefficient of about unity. Helium filling the open regions of the irregular structure then would lead to a shorter lifetime in a similar way as electrons do in metallic glasses. ${ }^{13}$ This will require a considerably higher intensity for saturation.

(ii) On the other hand, the helium atoms may directly form two-level systems if the irregular van der Waals potential of the hydrocarbons induces an irregular (glassy) structure in the atomic layers in helium next to them. Then the coupling of the phonons in the solid to the two-level states may be due to a modulation of the irregular van der Waals potential.

Our experiment does not yet allow us to distinguish between these different possible origins of the two-level systems. Yet the present results provide clear evidence for the first time* that saturable states can really exist and, furthermore, can really be responsible for the anomalous Kapitza conductance, at least in the special system studied here.

\section{REFERENCES}

1. L. J. Challis, J. Phys. C 7, 481 (1974).

2. H. Kinder and W. Dietsche, Phys. Rev. Lett. 33, 578 (1974); W. Dietsche and H. Kinder, J. Low Temp. Phys. 23, 27 (1976); J. Weber, W. Dietsche, and H. Kinder, Phys. Lett. 64A, 202 (1977).

3. W. Dietsche and H. Kinder, in Proc. of the 3rd Int. Conf. on Phonon Scattering in Condensed Matter (1979), to be published.

4. P. W. Anderson, B. I. Halperin, and C. Varma, Phil. Mag. 25, 1 (1972); W. A. Phillips, J. Low Temp. Phys. 7, 351 (1972).

5. W. Kaiser and M. Maier, in Laser Handbook, F. T. Arecci and E. O. Schulz-Dubois, eds. (North-Holland, Amsterdam, 1972), Vol. 2, p. 1077.

6. D. Pohl, M. Maier, and W. Kaiser, Phys. Rev. Lett. 20, 366 (1968).

7. H. E. Bömmel and K. Dransfeld, Phys. Rev. 117, 1245 (1960).

8. E. S. Sabisky and C. H. Anderson, Solid State Comm. 17, 1095 (1975).

9. W. Kappus and O. Weis, J. Appl. Phys. 44, 1947 (1973).

10. A. C. Anderson and W. L. Johnson, J. Low Temp. Phys. 7, 1 (1972).

11. J. Weber, W. Sandmann, W. Dietsche, and H. Kinder, Phys. Rev. Lett. 40, 1469 (1978).

12. S. Hunklinger and W. Arnold, in Physical Acoustics, Vol. 12, R. N. Thursten and W. P. Mason, eds. (Academic Press, New York, 1976), p. 155.

13. B. Golding, J. E. Graebner, A. B. Kane, and J. L. Black, Phys. Rev. Lett. 41, 1487 (1978).

14. K. C. Rawlings and J. C. A. van der Sluijs, J. Low Temp. Phys. 33, 313 (1978).

*The reduction of the Kapitza conductance reported by Rawlings and van der Sluijs ${ }^{14}$ (Cheeke effect) was only observed for "pulses" longer than $20 \mathrm{sec}$. Therefore, its origin must be totally different from the saturation obtained here with $10^{-7}$-sec pulses. 